\title{
Robust and Reliable Multidiscipline Ship Design
}

\author{
Shari E. Hannapel ${ }^{1}$ and Nickolas Vlahopoulos ${ }^{2}$ \\ University of Michigan, Ann Arbor, MI, 48105
}

\begin{abstract}
Mathematical formulations of reliability-based design and robust design have been developed for addressing the presence of uncertainty during optimization. Reliability addresses the effects of uncertainty on the constraints; constraints that are influenced by uncertainty are converted into new probabilistic constraints to ensure that the optimal solution will satisfy the constraints within a prescribed reliability level. Robustness is introduced by including the effects of the mean and variance of the response of the objective function in the optimization. This paper presents a formulation for applying reliabilitybased design and robust design within a network of parallel optimizations to account for uncertainty in multidiscipline design optimization. The method for multidiscipline design optimization under uncertainty is applied to a conceptual ship design with a model of a bulk carrier from the literature. The optimization results demonstrate that reliable and robust optimization can be applied effectively to ship design, and that it is important to consider uncertainty during the multidiscipline ship design process.
\end{abstract}

\section{Nomenclature}

$\alpha$
$\beta_{i}$
$\mathbf{d}$
$\mathbf{\sigma}$
$\boldsymbol{\delta}$
$f$
$F_{i}$
$G_{i}$
$\mathbf{g}_{i}$
$\mathbf{g}_{T}$
$\mu_{f}$
$\boldsymbol{\mu}_{p}$
$\boldsymbol{\mu}_{x}$
$N_{j}$
$O_{i}{ }^{\text {optimal }}$
$O_{i}{ }^{\text {start }}$
$O_{T}$
$P$
$\mathbf{p}^{2}$
$\sigma_{f}{ }^{2}$
$\mathbf{x}^{\text {current }}$
$\mathbf{x}_{i}{ }^{\text {start }}$
$\mathbf{x}_{i}{ }^{\text {optimal }}$
$x_{j, i}{ }^{\text {target }}$
$\mathbf{x}_{i}$
$\mathbf{x}_{T}$

$=$ weighting parameter for robust objective function

$=$ target reliability index for constraint $i$

$=$ vector of deterministic design variables

$=$ vector of standard deviations for design variables and parameters with uncertainty

$=$ vector of variation in design variables due to uncertainty

$=$ generic objective function

$=$ generic helpful function evaluated in discipline $i$

$=$ generic constraint

$=$ inequality constraints for the $i^{\text {th }}$ discipline

$=$ inequality constraints for the top level

$=$ mean of objective function $f$ under uncertainty

$=$ vector of mean values of parameters with uncertainty

$=$ vector of mean values of design variables

$=$ number of disciplines that share the $j^{\text {th }}$ design variable

$=$ optimal value for the objective function of the $t^{\text {th }}$ discipline

$=$ starting value for the objective function of the $i^{\text {th }}$ discipline

$=$ original top level objective function

$=$ probability distribution function

$=$ vector of parameters with uncertainty

$=$ variance of objective function $f$ under uncertainty

$=$ generic vector of design variables

$=$ vector of current values of design variables for the $i^{\text {th }}$ discipline

$=$ vector of starting values of design variables for the $i^{\text {th }}$ discipline

$=$ the optimal value of design variable $x_{j}$ in the $i^{\text {th }}$ discipline

$=$ target value for the $j^{\text {th }}$ design variable

$=$ vector of design variables for the $i^{\text {th }}$ discipline

$=$ vector of design variables for the top level

\footnotetext{
${ }_{1}^{1}$ Student, Department of Naval Architecture and Marine Engineering, 2600 Draper Dr., Ann Arbor, MI 48109.

${ }^{2}$ Professor, Department of Naval Architecture and Marine Engineering, 2600 Draper Dr., Ann Arbor, MI 48109.
} 


\section{Introduction}

I $\mathrm{N}$ design optimization, the optimal solution is a single point that minimizes (or maximizes) a function of interest while satisfying a prescribed set of constraints. Often, the effects of uncertainty are not considered during the ship design optimization process. However, in real life, design variables or environmental parameters contain uncertainty; for example, manufacturing tolerances or environmental conditions are beyond the control of the designer, but they do affect the performance of the design. Further, the simulation tools or the regression models which provide performance information for a ship during the decision-making process are additional sources of uncertainty because of their inherent variability in the predictions. Due to the variations in the uncertain design variables and parameters, or due to the uncertainty in the simulation models, the response of the optimal design will differ from the deterministic expectation. This can lead to violation of the active constraints and/or deterioration of the expected optimal performance. Capturing such effects within the early stages of the ship design process will eliminate expensive design modifications at later stages.

Multidisciplinary design optimization (MDO) describes the process of dividing the design of a system into disciplines and seeking a solution which satisfies all discipline-level constraints, improves the discipline level objectives as much as possible, and improves the overall system level objective. MDO can be a valuable tool for the design of any complex system which features competing disciplines; MDO has previously been applied to the design of an aircraft wing, an undersea vehicle, and to preliminary ship design ${ }^{1-3}$, among many examples in the literature.

In reliability-based design, the effects of uncertainties in design variables and parameters on the constraints are considered. When reliability is introduced in a design process, the constraints which are influenced by uncertainty are converted into probabilistic constraints. This ensures that the optimal solution will satisfy the constraints in the presence of uncertainty within a prescribed reliability level. Methods for accounting for uncertainty in the constraints have been shown in References 4 and 5. Many applications of reliability-based design can be found in the literature. References 6 and 7 apply reliability-based design to vehicle design for crashworthiness; Reference 8 applies reliability-based design to an airplane wing structure; Reference 9 uses reliability-based design for structural design subject to earthquake loading; and Reference 10 applies reliability-based design to the design of an exhaust system. Additionally, reliability-based design optimization has been applied to multidisciplinary design optimization in References 11-13.

In robust design optimization, the goal is to minimize not only the objective function but also the variation in the objective function about the optimum point. Then a new robust objective function is formulated as a function of the mean and variance of the response of the objective function at a selected design point. Reference 14 provides a thorough summary of methods for applying robust optimization. Many applications of robust design optimization can be found in the literature. In Reference 15, robust optimization is applied to the design of layered plate bonding; Reference 16 uses a robust design strategy with a snap-fit device; Reference 17 applies robust optimization to an airplane component; and Reference 18 introduces robustness in optimizing the design of a transistor device. Robust optimization has also been applied to multidisciplinary design problems. Reference 19 uses robust optimization with a multi-objective design application to a V6 engine; Reference 20 applies a multi-objective evolutionary algorithm for robust design of a welded beam and a bulk carrier; and Reference 21 uses multidisciplinary robust design for chemical filtration optimization.

This paper introduces a formulation for multidisciplinary design optimization under uncertainty that includes principles of reliability-based design and robust design within a network of parallel optimizations. The theoretical background is presented first, including the multidisciplinary design optimization method for accounting for the effect of uncertain design variables and parameters on the constraints. Methods for introducing a robust objective function to the multidisciplinary design optimization are also discussed.

The main new element introduced by this paper is the combination of multidisciplinary design optimization which takes into account both reliability and robustness in the solution. Additionally, this new approach is applied to the concept design of a bulk carrier to demonstrate how uncertainty can be accounted for in multidisciplinary ship design.

\section{Multidiscipline Design Optimization}

The mathematical background of the method which is employed in this work for driving the network of optimizations and coordinating the exchange of information is presented in this section. The optimization of several disciplines is coordinated through a top level optimization, which is typically selected as a discipline of a global nature (such as cost). Discipline level constraints are also enforced in the top level, ensuring that the final solution satisfies all of the constraints. Discipline optimizations explicitly influence the overall direction of the system 
solution based on the improvement encountered at the discipline level. An additional feature of this algorithm is that different optimization methods can be used for the system and discipline level optimizations.

At the beginning of each iteration of the top level optimization, individual discipline-level optimizations are performed using the values of the design variables from the previous step of the top level optimization as the starting values. After the discipline level optimizations are complete, a group of target values for the design variables are determined based on the improvement in the discipline level objectives:

$$
x_{j}^{\text {target }}=\frac{\sum_{i=1}^{N_{j}}\left(x_{j, i}{ }^{\text {optimal }}\left|O_{i}^{\text {start }}-O_{i}^{\text {optimal }}\right|\right)}{\sum_{i=1}^{N_{j}}\left|O_{i}^{\text {start }}-O_{i}^{\text {optimal }}\right|}
$$

In Eq. (1), $x_{j}^{\text {target }}$ is the target value for the $j^{\text {th }}$ design variable; $x_{j, i}^{\text {optimal }}$ indicates the optimal value of design variable $x_{j}$ in the $i^{\text {th }}$ discipline. The objective function of the $i^{\text {th }}$ discipline is $O_{i}$ where the superscripts start and optimal indicate starting and optimal values the objective functions. $N_{j}$ is the total number of disciplines that share the $j^{\text {th }}$ design variable.

The overall top level objective function can be stated as

$$
\min \left(O_{T}+\sum_{j=1}^{J}\left|x_{j}^{\text {target }}-x_{j}\right|\right)
$$

where $O_{T}$ is the original top level objective function and $J$ is the total number of design variables in the top level. The effects of the discipline objective functions are included by requiring that the top level design variables match the target values, and at the same time the top level objective function is improved.

All of the discipline-level constraints are included in the top level optimization along with the top level constraints; this ensures that the optimal point will also be a feasible point for all of the disciplines. The constraints are expressed mathematically as

$$
\begin{aligned}
\mathbf{g}_{T}\left(\mathbf{x}_{T}\right) & \leq 0 \\
\mathbf{g}_{i}\left(\mathbf{x}_{i}\right) & \leq 0
\end{aligned}
$$

where $\mathbf{g}_{T}$ are the inequality constraints for the top level and $\mathbf{g}_{i}$ are the inequality constraints for the $i^{\text {th }}$ discipline. The vector of design variables for the top level objective is $\mathbf{x}_{T}$ and the vector of design variables for the $i^{\text {th }}$ discipline is $\mathbf{x}_{i}$.

The MDO algorithm is illustrated in Fig. 1. The top level coordinates information about the top level objective, the top and discipline level design variables, and the top and discipline level constraints with the information from the discipline level optimizations. The algorithm also includes the capability to use compute (optional) additional useful functions $F_{i}$ that may be used in the evaluation of the objective functions.

\section{Reliability-Based Design}

The multidisciplinary optimization framework used in this work is also capable including the effect of uncertainty in design variables and/or parameters on the constraints. The framework uses a single-loop reliability-based design optimization (RBDO) algorithm ${ }^{22,23}$.

The single-loop RBDO algorithm converts the probabilistic optimization problem into an equivalent deterministic problem. Then the single-loop RBDO problem for one objective function $f$ and the accompanying set of constraints can be stated as:

$$
\begin{gathered}
\min _{\mathbf{x}, \boldsymbol{\mu}_{x}} f\left(\mathbf{d}, \boldsymbol{\mu}_{x}, \boldsymbol{\mu}_{p}\right) \\
\text { subject to: } \quad G_{i}\left(\mathbf{d}, \mathbf{X}_{i}, \mathbf{p}_{i}\right) \leq 0 \quad i=1, \ldots, n
\end{gathered}
$$




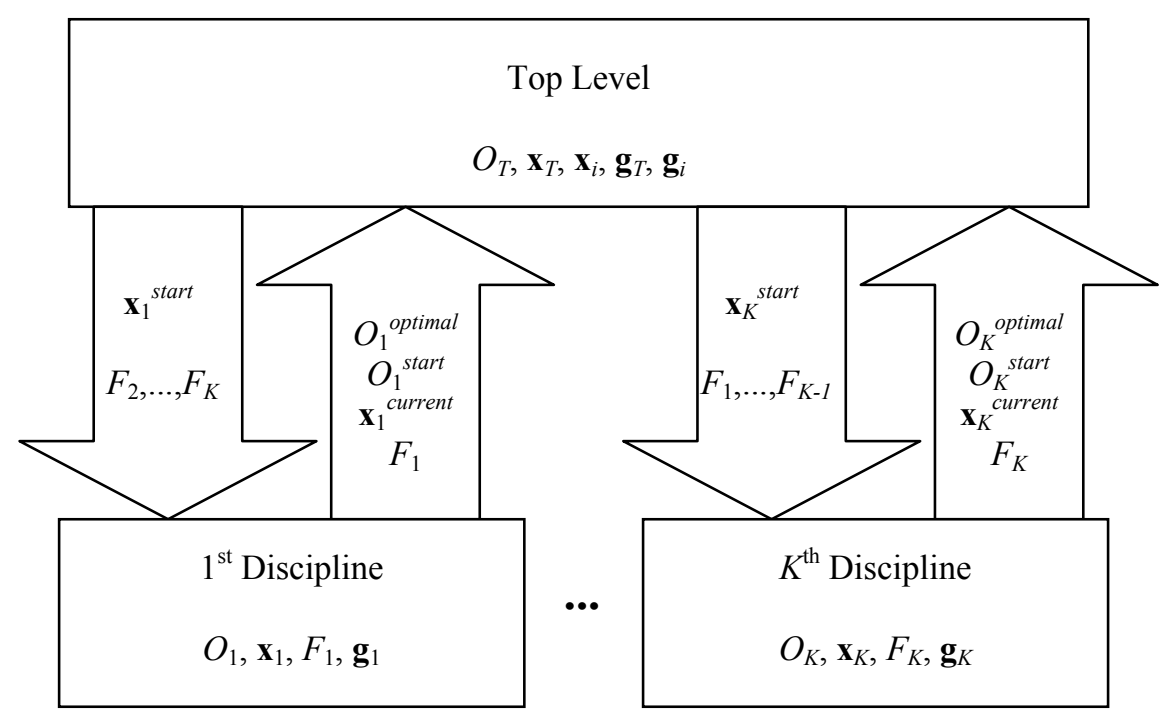

Figure 1. Flow chart of the multidisciplinary design optimization algorithm.

where $f$ is the objective function, $\mathbf{x}$ is the vector of deterministic design variables, $\boldsymbol{\mu}_{x}$ is the vector of the mean values of the random design variables, and $\boldsymbol{\mu}_{p}$ is the vector of the mean values of the parameters. The $n$ constraints $G_{i}$ are functions of $\mathbf{d}$ and the quantities:

$$
\begin{gathered}
\mathbf{X}_{i}=\boldsymbol{\mu}_{x}-\boldsymbol{\sigma} \beta_{i} \alpha_{i} \\
\mathbf{p}_{i}=\boldsymbol{\mu}_{p}-\boldsymbol{\sigma} \beta_{i} \alpha_{i} \\
\alpha_{i}=\frac{\boldsymbol{\sigma} \cdot \nabla G_{i}\left(\mathbf{d}, \mathbf{X}_{i}, \mathbf{p}\right)}{\left\|\boldsymbol{\sigma} \cdot \nabla G_{i}\left(\mathbf{d}, \mathbf{X}_{i}, \mathbf{p}\right)\right\|} \\
\mathbf{d}^{l} \leq \mathbf{d} \leq \mathbf{d}^{u} \\
\boldsymbol{\mu}_{x}{ }^{l} \leq \boldsymbol{\mu}_{x} \leq \boldsymbol{\mu}_{x}{ }^{u}
\end{gathered}
$$

The target reliability index for the $i^{\text {th }}$ constraint is $\beta_{i}$, which describes the desired confidence that a constraint will be satisfied. $\alpha_{i}$ is the normalized gradient of the $i^{\text {th }}$ constraint, and $\sigma$ is the vector of standard deviations for the design variables and parameters. Equations (9) and (10) describe the upper and lower bounds on the design variables and the mean values.

The objective function is evaluated at the mean point $\left(\mathbf{d}, \boldsymbol{\mu}_{x}, \boldsymbol{\mu}_{p}\right)$, and the constraints are evaluated at the deterministic optimum. This introduces a safety margin in each constraint determined by the target reliability index and the rate of change of the constraint function.

\section{Robust Optimization}

In this work, the robust objective function $R_{f}$ for an objective function $f$ is defined as in Refs. 14 and 19, using a weighted-sum approach:

$$
R_{f}(\mathbf{x})=\alpha \mu_{f}(\mathbf{x})+(1-\alpha) \sigma_{f}^{2}(\mathbf{x})
$$

where $\mu_{f}(\mathbf{x})$ and $\sigma_{f}^{2}(\mathbf{x})$ are the mean and variance of the response of $f$ at the design point $\mathbf{x}$, where $\alpha$ is a weighting parameter, $0 \leq \alpha \leq 1$, that can be adjusted for the relative importance of the mean and variance for the particular application. 
The following equations (from Ref. 14) can be used to calculate the mean and variance of the objective function $f$ that are needed for Eq. (11):

$$
\begin{gathered}
\mu_{f}(\mathbf{x})=\int f(\mathbf{x}+\boldsymbol{\delta}, \mathbf{p}) P(\boldsymbol{\delta}, \mathbf{p}) d \boldsymbol{\delta} d \mathbf{p} \\
\sigma_{f}{ }^{2}(\mathbf{x})=\int(f(\mathbf{x}+\boldsymbol{\delta}, \mathbf{p}))^{2} P(\boldsymbol{\delta}, \mathbf{p}) d \mathbf{\delta} d \mathbf{p}-\left(\mu_{f}(\mathbf{x})\right)^{2}
\end{gathered}
$$

In Eqs. (12) and (13), the design variables $\mathbf{x}$ are assumed to be deterministic, but with a random variation term $\boldsymbol{\delta}$; that is, the probabilistic design variables can be described by $\mathbf{x}+\boldsymbol{\delta}$. $\mathbf{p}$ is the vector of random parameters, and $P(\boldsymbol{\delta}, \mathbf{p})$ is the joint probability distribution function for $\boldsymbol{\delta}$ and $\mathbf{p}$.

\section{Bulk Carrier Model}

Reference 24 presents the preliminary design of a bulk carrier using simple empirical models. The optimization of this preliminary design has been studied in the literature, including using a multi-objective design optimization approach $^{25}$; implementing a particle swarm optimizer ${ }^{1}$; and applying neural networks to reduce computational effort ${ }^{20}$.

The ship is described by six design variables: length $L$, beam $B$, depth $D$, draft $T$, block coefficient $C_{B}$, and speed $V_{k}$. The design variables are used to evaluate the three objective functions: lightship weight, annual cargo, and transportation cost. Details for the calculation of the objective functions are included in Table 1. Table 1 shows the objective functions, design, variables, constraints, and other formulas used in the calculations.

The notation in Table 1 has been modified slightly from Ref. 24. It is worth noting that the empirical constraint for stability listed in Table 1 comes from the empirical formula and constraint in Ref. 24 for GM; the empirical expression for GM is given by

$$
G M=0.53 T+\frac{\left(0.085 C_{B}-0.002\right) B^{2}}{T \cdot C_{B}}-1.0-0.52 D
$$

and the requirement on $G M$ for stability is

$$
G M \geq 0.07 B
$$

\section{Application to the Bulk Carrier Example}

The new formulation that integrates algorithms for multidiscipline design, reliability-based design, and robust design is applied to the preliminary design for the bulk carrier. The transportation cost was the system level optimization, while the lightship weight and annual cargo comprise the discipline level optimizations. The goal is to minimize the transportation cost and the lightship weight while maximizing the annual cargo. (Note that the optimizer only seeks to minimize each objective function, so the negative of the annual cargo is minimized.)

The initial point for the optimization analysis was $\left(L, B, D, T, C_{B}, V_{k}\right)=(195,32.31,20,10.5,0.7,16)$, because this point satisfies all of the constraints and has been used in previous work with the bulk carrier model. The objective functions were scaled by their values at this initial point to prevent the very large values (order $10^{5}$ ) of the annual cargo from dominating over the smaller (order $10^{\circ}$ ) values of the transportation cost. The values of the objective functions at the initial point are: 10,303 t lightship weight; 547,860 t/yr annual cargo; and 9.926 £/t transportation cost. Multiple analyses were performed in order to compare deterministic and probabilistic results, and for investigating the effects of alternative robustness definitions on the results. 


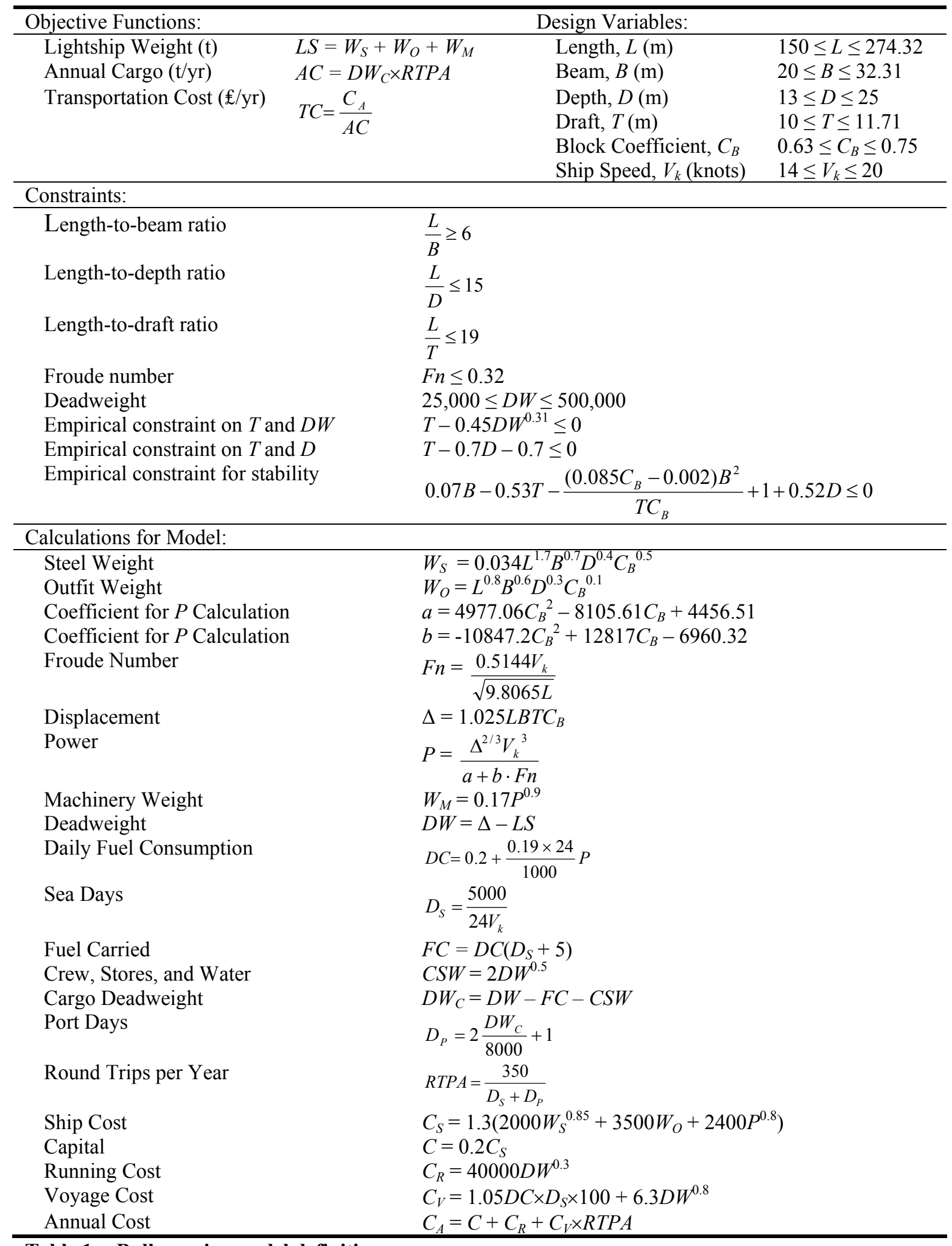

Table 1. Bulk carrier model definition. 


\section{A. MDO without Uncertainty}

The multidisciplinary optimization was run for the bulk carrier example using the objective functions and constraints listed in Table 1, without including uncertainty in any of the design variables or parameters. This was done to give a baseline for comparison to later results where uncertainty is considered. The results are listed in Table 2 in the column labeled Deterministic Optimum.

\begin{tabular}{lrrr}
\hline & $\begin{array}{c}\text { Deterministic } \\
\text { Optimum }\end{array}$ & $\begin{array}{c}\text { Reliable } \\
\text { Optimum }\end{array}$ & $\begin{array}{c}\text { Reliable \& } \\
\text { Robust Optimum }\end{array}$ \\
\hline Transportation Cost & 8.424 & 8.635 & 8.838 \\
Annual Cargo & 506,320 & 550,140 & 566,690 \\
Lightship Weight & 8,198 & 8,803 & 8,462 \\
\hline$L$ & 188.90 & 193.86 & 182.61 \\
$B$ & 31.485 & 32.310 & 30.435 \\
$D$ & 15.729 & 15.364 & 15.729 \\
$T$ & 11.710 & 11.455 & 11.710 \\
$C_{B}$ & 0.630 & 0.657 & 0.750 \\
$V_{k}$ & 14.000 & 15.027 & 15.027 \\
\hline
\end{tabular}

Table 2. Summary of results from multiple MDO analyses.

\section{B. MDO with Reliability-Based Design}

Uncertainty was introduced into the bulk carrier model in two ways: uncertainty in a design variable and uncertainty in a parameter. Uncertainty in a design variable was introduced for the ship speed. For a selected design ship speed $V_{\text {design }}$, the actual ship speed follows a normal distribution about $V_{\text {design }}$; that is,

$$
V_{\text {actual }}=V_{\text {design }}+v
$$

where $V_{\text {actual }}$ is the actual ship speed and $v$ is a normally distributed random variable with mean zero; the uncertainty in the ship speed was defined in this manner to agree with the form of Eqs. (12) and (13). Equation (15) describes the physical situation that the intended ship speed is $V_{\text {design }}$, but due to sea conditions the actual ship speed varies by the amount $v$. For this analysis, $v$ follows a normal distribution with mean zero and standard deviation of 0.5 knots.

Next, uncertainty in a parameter was introduced in the model. The model defined by Reference 24 as given in Table 1 uses the following regression equation to calculate the steel weight, $W_{S}$, of the ship:

$$
W_{S}=0.034 L^{1.7} B^{0.7} D^{0.4} C_{B}^{0.5}
$$

To apply uncertainty in a parameter to this model, the exponent on the ship length $L$ is considered to be a random parameter, $\varepsilon$ :

$$
W_{S}=0.034 L^{\varepsilon} B^{0.7} D^{0.4} C_{B}{ }^{0.5}
$$

This uncertain parameter can be used to describe uncertainty in the model itself and to illustrate the effects of any modeling inaccuracies. For this analysis, the parameter $\varepsilon$ follows a normal distribution with mean 1.7 and standard deviation 0.05 .

There are five total constraints that are affected by these uncertainties. The simplest constraints are the upper and lower limits on the ship speed. The other constraints affected by the uncertainties (repeated from Table 1) are the constraints on Froude number $F n$ and deadweight $D W$ :

$$
\begin{gathered}
F n \leq 0.32 \\
25,000 \leq D W \leq 500,000 \\
T-0.45 D W^{0.31} \leq 0
\end{gathered}
$$


The multidisciplinary optimization was run including the uncertainty in the constraints (where applicable) and the results are included in Table 2. A 98\% reliability level was prescribed for the probabilistic constraints. It is immediately clear that the performance in all three objectives at this selected optimum is inferior to the performance at the deterministic optimum. This is expected because a safety margin has been introduced in order to satisfy the constraints in the presence of uncertainty. The constraints were evaluated at the optimum point and the values are shown in Table 3; note that the constraints that are affected by uncertainty are indicated with a check mark in the column labeled Uncertainty. Two constraints are active, but the three constraints which are affected by the uncertainty have an appropriate margin to account for the variability in the ship speed $V_{k}$ and exponent $\varepsilon$.

\begin{tabular}{lcc}
\hline Constraint & Constraint Value & Uncertainty \\
\hline$\frac{L}{B} \geq 6$ & 6.000 & \\
$\frac{L}{D} \leq 15$ & 12.6180 & \\
$\frac{L}{T} \leq 19$ & 16.9241 & \\
$F n \leq 0.32$ & 0.1773 & $\checkmark$ \\
$25,000 \leq D W \leq 500,000$ & 39,435 & $\checkmark$ \\
$T-0.45 D W^{0.31} \leq 0$ & -0.5110 & $\checkmark$ \\
$T-0.7 D-0.7 \leq 0$ & $-2.0206 \times 10^{-14}$ & \\
$0.07 B-0.53 T-\frac{\left(0.085 C_{B}-0.002\right) B^{2}}{T C_{B}}+1+0.52 D \leq 0$ & -2.2892 & \\
\hline
\end{tabular}

Table 3. Constraint evaluation using reliability-based design optimization.

\section{MDO with Uncertainty in Constraints and Objective Functions}

A slightly modified form of Eq. (11) (similar to that of Reference 19) is used to apply robustness to the bulk carrier example for each objective function $f$ :

$$
R_{f}\left(L, B, D, T, C_{B}, V_{k}\right)=\alpha \frac{\mu\left(L, B, D, T, C_{B}, V_{k}\right)}{f_{0}}+(1-\alpha) \frac{\sigma\left(L, B, D, T, C_{B}, V_{k}\right)}{\sigma_{0}}
$$

The mean $\mu$ and variance $\sigma$ are evaluated at the design point $\left(L, B, D, T, C_{B}, V_{k}\right)$. The variance is scaled by $\sigma_{0}$, the value of the variance at the initial point. The mean is scaled by $f_{0}$, the value of the objective function $f$ at the initial point, instead of the mean value at the initial point in order to allow comparison between the robust optimization results and the other results.

To calculate the necessary mean and standard deviation, Equations (12) and (13) are used with the two random variables $v$ and $\varepsilon$. Then the equations become:

$$
\begin{gathered}
\mu\left(L, B, D, T, C_{B}, V_{k}\right)=\iint f\left(L, B, D, T, C_{B}, V_{k}+v, \varepsilon\right) p(v, \varepsilon) d v d \varepsilon \\
\sigma^{2}\left(L, B, D, T, C_{B}, V_{k}\right)=\iint\left(f\left(L, B, D, T, C_{B}, V_{k}+v, \varepsilon\right)\right)^{2} p(v, \varepsilon) d v d \varepsilon-\left(\mu\left(L, B, D, T, C_{B}, V_{k}\right)\right)^{2}
\end{gathered}
$$

where $f$ is any of the objective functions and $p(v, \varepsilon)$ is the joint probability distribution function for $v$ and $\varepsilon$. The ship speed variation and the exponent are assumed to be independent, so the equations become

$$
\begin{gathered}
\mu\left(L, B, D, T, C_{B}, V_{k}\right)=\iint f\left(L, B, D, T, C_{B}, V_{k}+v, \varepsilon\right) p_{v}(v) p_{\varepsilon}(\varepsilon) p d v d \varepsilon \\
\sigma^{2}\left(L, B, D, T, C_{B}, V_{k}\right)=\iint\left\{f\left(L, B, D, T, C_{B}, V_{k}+v, \varepsilon\right)\right\}^{2} p_{v}(v) p_{\varepsilon}(\varepsilon) d v d \varepsilon-\left\{\mu\left(L, B, D, T, C_{B}, V_{k}\right)\right\}^{2}
\end{gathered}
$$

where $p_{v}(v)$ and $p_{\varepsilon}(\varepsilon)$ are the probability distribution functions for $v$ and $\varepsilon$, respectively. 
The integrals are evaluated numerically during the optimization. Each PDF is integrated over $\pm 4 \sigma$ to cover approximately $99.99 \%$ of the area under the normal curve. The numerical integration is performed using 200 points in each random variable, or 40,000 points total over the integrand. The effect of increasing or decreasing the number of points was investigated and it was found that 200 points led to a satisfactory compromise of computing time and accuracy. Additionally, the results of the integration were compared to the results of a simple Monte Carlo simulation, which randomly placed 1000 points in the design space according to the distributions of $v$ and $\varepsilon$. The mean and variance resulting from the Monte Carlo simulations confirmed the results of the numerical integration.

The robust and reliable optimization was performed using the parameter value $\alpha=0.5$; the results are included in Table 2. The constraints at the robust optimum were evaluated and are included in Table 4. All of the constraints are satisfied, with two of the constraints active: the constraint on the length-to-beam ratio and the empirical constraint on the draft and depth. The safety margins introduced in the constraints are comparable to the margins in the probabilistic constraints of the MDO reliability analysis. However, the margins differ because in the current case the optimum has shifted due to the robust considerations.

\begin{tabular}{lrr}
\hline \multicolumn{1}{c}{ Constraint } & Constraint Value & Uncertainty \\
\hline$\frac{L}{B} \geq 6$ & $4.7071 \times 10^{-6}$ & \\
$\frac{L}{D} \leq 15$ & 11.6102 & \\
$\frac{L}{T} \leq 19$ & & \\
$F n \leq 0.32$ & 15.5946 & \\
$25,000 \leq D W \leq 500,000$ & 0.1827 & $\checkmark$ \\
$T-0.45 D W^{0.31} \leq 0$ & 41,569 & $\checkmark$ \\
$T-0.7 D-0.7 \leq 0$ & -0.4528 & $\checkmark$ \\
$0.07 B-0.53 T-\frac{\left(0.085 C_{B}-0.002\right) B^{2}}{T C_{B}}+1+0.52 D \leq 0$ & $-2427 \times 10^{-14}$ & \\
\end{tabular}

Table 4. Constraint evaluation using reliable and robust optimization.

To study the effects of the robust optimization on the optimum design, the statistics at the three different optima were computed; Table 5 summarizes the statistics for the three optimization approaches. The deterministic optimum shows the best performance for the transportation cost (the top level objective) and the lightship weight. However, for the deterministic case, without the consideration of uncertainty in the constraints, it is unlikely that the constraints would be satisfied when considering the uncertainty of the ship speed $V_{k}$ and exponent $\varepsilon$. For the robust and reliable case, the standard deviation has been reduced from the reliable case for all three of the objective functions.

\begin{tabular}{lrrr}
\hline & $\begin{array}{c}\text { Deterministic } \\
\text { Optimum }\end{array}$ & $\begin{array}{c}\text { Reliable } \\
\text { Optimum }\end{array}$ & $\begin{array}{c}\text { Robust \& Reliable } \\
\text { Optimum }(\alpha=0.5)\end{array}$ \\
\hline Transportation Cost & 8.4240 & 8.6351 & 8.8380 \\
Transportation Cost Mean & 8.5401 & 8.7523 & 8.9472 \\
Transportation Cost Standard Deviation & 0.7174 & 0.7241 & 0.6792 \\
Annual Cargo & 506,320 & 550,140 & 566,690 \\
Annual Cargo Mean & 503,650 & 547,300 & 564,160 \\
Annual Cargo Standard Deviation & 20,504 & 21,022 & 19,266 \\
Lightship Weight & 8,198 & 8,803 & 8,462 \\
Lightship Weight Mean & 8,434 & 9,137 & 8,698 \\
Lightship Weight Standard Deviation & 1,858 & 2,012 & 1,857 \\
\hline
\end{tabular}

Table 5. Statistics for the optimization results.

To illustrate the effect of selecting a reliable optimum, a Monte Carlo simulation was performed to generate random realizations of the ship design about the optimum design values. For the deterministic optimum design $(188.90,31.485,15.729,11.710,0.630,14.000)$, only 2424 out of 10,000 randomly generated points satisfied all of 
the constraints in the presence of uncertainty. For the reliable optimum design $(193.86,32.310,15.364,11.455$, $0.657,15.027), 9574$ out of 10,000 randomly generated points satisfied all of the constraints in the presence of uncertainty; this is a very significant improvement over the deterministic case. For the reliable and robust optimum design $(182.61,30.435,15.729,11.710,0.750,15.027), 9595$ out of 10,000 randomly generated points satisfied all of the constraints in the presence of uncertainty. The results for the robust and robust and reliable optima are reasonable for the $98 \%$ reliability used in the analysis.

To visualize the results, the values of the objective functions at the optima can be plotted. A simple Monte Carlo technique was applied to approximate the Pareto front in the three-dimensional plot (this method was selected for convenience, since the purpose of this work was not to find the Pareto front). The optima are shown in two twodimensional plots in Fig. 2: the deterministic optimum is shown in blue, the reliable optimum is shown in red, and the robust and reliable optimum is shown in green.

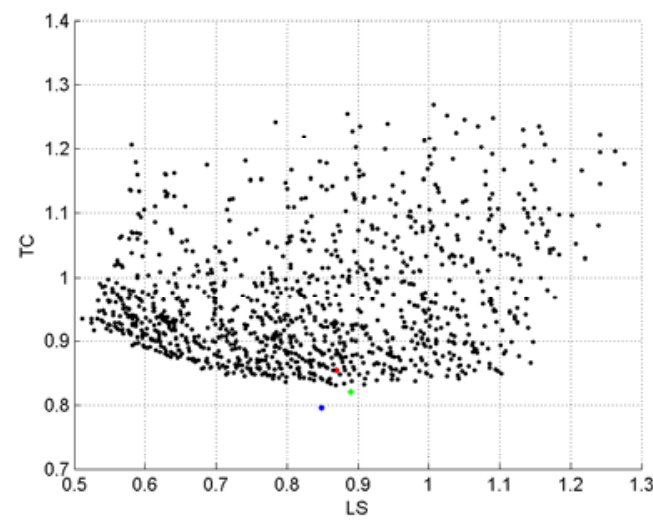

(a)

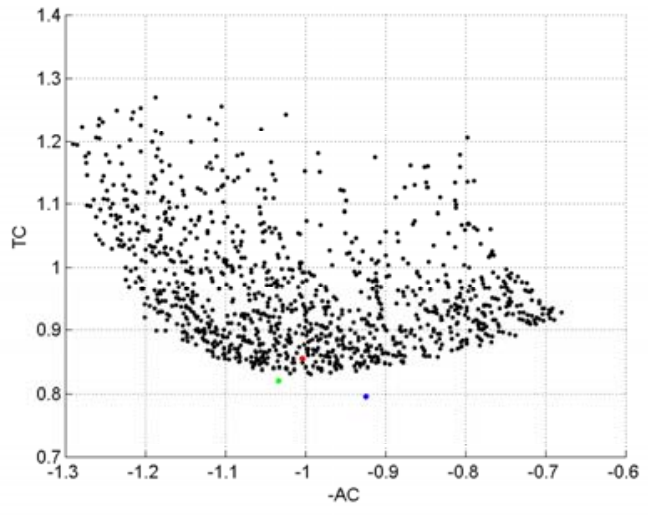

(b)

Figure 2. Approximated Pareto front plots with optima.

In Fig. 2, the objective functions are scaled so that all of the objective functions have a magnitude of one at the initial point. Fig. 2(a) shows the scaled lightship weight (LS) versus the scaled transportation cost (TC), and Fig. 2(b) shows the negative of the scaled annual cargo (-AC) versus the scaled transportation cost. Note that for this problem which has three objective functions, the Pareto front would be represented by a surface; the twodimensional plots in Fig. 2 effectively show a projection of an approximation of the surface.

Figure 2 shows not only that the new MDO algorithm is selecting points that perform relatively well, but also that the algorithm seeks to reduce the top level objective as much as possible (transportation cost) while the discipline level objectives are not as important.

\section{Conclusion}

Methods of reliability-based design and robust optimization have been applied to a multidiscipline design optimization framework. The multidiscipline design optimization is applied to the conceptual design of a bulk carrier using a model from the literature. The results for the optimum ship design are compared for cases which do not consider uncertainty and for cases which consider reliable optimization and reliable and robust optimization.

\section{Acknowledgments}

The authors would like to thank the SMART Scholarship Program.

\section{References}

${ }^{1}$ Hart, C., Vlahopoulos, N., "An Integrated Multidisciplinary Particle Swarm Optimization Approach to Conceptual Ship Design," Structural and Multidisciplinary Optimization, Vol. 41, No. 3, pp. 481-494.

${ }^{2}$ He, J., Vlahopoulos, N., "Utilization of Response Surface Methodologies in the Multi-discipline Design Optimization of an Aircraft Wing," SAE Paper 2009-01-0344, 2009.

${ }^{3}$ He, J., Zhang, G., Vlahopoulos, N., "Uncertainty Propagation in Multi-Disciplinary Design of Undersea Vehicles,” SAE Paper 2008-01-0128, 2008. 
${ }^{4}$ Sun, J., Zhang, G., Vlahopoulos, N., Hong, S.B., "Development of Multi-Disciplinary Optimization under Uncertainty for Thermal Protection Systems Applications," 11th AIAA/ISSMO Multidisciplinary Analysis and Optimization Conference, Sept. 2006.

${ }^{5}$ Vlahopoulos, N., He, J., “Application of a Multidiscipline Design Optimization Approach for Designing the Thermal Protection System of an Apollo Type Vehicle Under Uncertainty," JANNAF Interagency Propulsion Committee 6th MSS, 4th LPS, 3rd SPS Joint Meeting, Dec., 2008.

${ }^{6}$ Acar, E., Solanki, K., "System Reliability Based Vehicle Design for Crashworthiness and Effects of Various Uncertainty Reduction Measures," Structual and Multidisciplinary Optimization, Vol. 39, pp. 311-325, 2009.

${ }^{7}$ Zou, T., Mahadevan, S., “A Direct Decoupling Approach for Efficient Reliability-Based Design Optimization,” Structual and Multidisciplinary Optimization, Vol. 31, pp. 190-200, 2006.

${ }^{8}$ Allen, M., Maute, K., "Reliability-Based Design Optimization of Aeroelastic Structures," Structural and Multidisciplinary Optimization, Vol 27, pp. 228-242, 2004.

${ }^{9}$ Lagaros, N. D., Papadrakakis, M., "Robust Seismic Design Optimization of Steel Structures," Structural and Multidisciplinary Optimization, Vol. 33, pp. 457-469, 2007.

${ }^{10}$ Yang, R. J., Chuang, C., Guo, L., Li, G., "Experience with Approximate Reliability-Based Optimization Methods II: An Exhaust System Problem," Structural and Multidisciplinary Optimization, Vol. 29, pp. 488-497, 2005.

Ahn, J., Kwon, J. H., "An Efficient Strategy for Reliability-Based Multidisciplinary Design Optimization Using BLISS" Structural and Multidisciplinary Optimization, Vol. 31, pp. 363-372, 2006.

${ }^{12}$ Chiralaksanakul, A., Mahadevan, S., "Decoupled Approach to Multidisciplinary Design Optimization under Uncertainty," Optimization and Engineering, Vol. 8, pp. 21-42, 2007.

${ }^{13}$ Du, X., Guo, J., Beeram, H., "Sequential Optimization and Reliability Assessment for Multidisciplinary Systems Design," Structural and Multidisciplinary Optimization, Vol. 35, pp. 117-130, 2008.

${ }^{14}$ Beyer, H. G., Sendhoff, B., "Robust Optimization - A Comprehensive Survey," Computer Methods in Applied Mechanics and Engineerng, Vol. 196, pp. 3190-3218, 2007.

${ }^{15}$ Choi, J. H., Lee, W. H., Park, J. J., Youn, B. D., “A Study on Robust Design Optimization of Layered Plate Bonding Process Considering Uncertainties,” Structural and Multidisciplinary Optimization, Vol. 35, pp. 531-540, 2008.

${ }^{16}$ Reale-Levis, F. D., Romero, V. J., Swiler L. P., “An Efficient Robust Design Strategy Employing Surrogate Variance Minimization and Adaptive Mean Constraint Satisfaction,” 2008 10th AIAA Non-Deterministic Approaches Conference, April, 2008.

${ }^{17}$ Steenackers, G., Guillaume, P., Vanlanduit, S., "Robust Optimization of an Airplane Component Taking into Account the Uncertainty of the Design Parameters," Quality and Reliability Engineering International, Vol. 25, pp. 255-282, 2009.

${ }^{18}$ Delpiano, J., Sepúlveda, M., "Combining Iterative Heuristic Optimization and Uncertainty Analysis Methods for Robust Parameter Design,” Engineering Optimization, Vol. 38, No. 7, pp. 821-831, 2006.

${ }^{19}$ Wang, W. M., Peng, Y. H., Hu, J., Cao, Z. M., “Collaborative Robust Optimization under Uncertainty Based on Generalized Dynamic Constraints Network,” Structural and Multidisciplinary Optimization, Vol. 38, pp. 159-170, 2009.

${ }^{20}$ Ray, T., Smith, W., “A Surrogate Assisted Parallel Multiobjective Evolutionary Algorithm for Robust Engineering Design,” Engineering Optimization, Vol. 38, No. 8, pp. 997-1011, 2006.

${ }^{21}$ Kovach, J., Cho, B. R., "Development of a Multidisciplinary-Multiresponse Robust Design Optimization Model," Engineering Optimization, Vol. 40, No. 9, pp. 805-819, 2008.

${ }^{22}$ Liang, J., Mourelatos, Z. P., Tu, J., “A Single-Loop Method for Reliability-Based Design Optimization,” International Journal of Product Development, Vol. 5, No. 1/2, pp. 76-92, 2008. 
${ }^{23}$ Mourelatos, Z. P., Vlahopoulos, N., Ebrat, O., Liang, J., Wang, J., "Probabilistic Main Bearing Performance for an Internal Combustion Engine," ASME Journal of Tribology, Vol. 127, No. 4, pp.784-792, 2005.

${ }^{24}$ Sen, P., Yang, J., Multiple Criteria Decision Support in Engineering Design, Springer, London, 1998, pp. 150-157.

${ }^{25}$ Parsons, M. G., Scott, R. L., "Formulation of Multicriterion Design Optimization Problems for Solution with Scalar Numerical Optimization Methods,” Journal of Ship Research, Vol. 48, No. 1, pp. 61-76, 2004. 